Peridotite boulders found interbedded with basalt at site 395 (Melson, W.G. et al. Init. Rep. DSDP Leg 45; 1978) suggests that the relationships observed at site 670 were common, at least over the past 7 million years. At present, talus moving down the wall of the median valley from the peridotite exposure is being periodically interbedded with basalt flows from the axis of the valley only a few kilometres away. Such areas of thin or missing layer 2 (and possibly missing layer 3 ) seem to be common at slow-spreading centres such as the Mid-Atlantic Ridge.

\title{
Neurology
}

\section{Pharmacological treatment of central nervous system injury}

\section{from Bernhard A. Sabel and Donald G. Stein}

UNTIL recently it was believed that brain and spinal cord injuries cannot be medically treated. Now, however, it seems that some of the secondary deterioration of damaged brain and spinal cord tissue can be reduced by new drugs*. These new approaches hold considerable promise for therapy and raise hopes that brain and spinal cord injury in humans can be repaired.

When central nervous system injury occurs, areas of the brain are lost, interrupting the circuitry of neuronal connections. Within minutes after the initial trauma, the remaining tissue starts to deteriorate further, characterized by the progressive death of partially damaged neurones near the lesion, apparently caused by a hostile biochemical environment. Current drug therapies are aimed at reducing these detrimental secondary events. The metabolites of free fatty acids contribute to the deterioration of the surviving tissue and there has been some success in reducing elevated prostaglandin and thromboxane levels in patients (Paul Demediuk, Veterans Administration Center, San Francisco).

Such pharmacological approaches are clinically successful only if the neurones spared by the initial trauma and saved from secondary cell death by drugs can maintain behavioural function. After spinal cord trauma, only 10 per cent of the axons in the ventral funiculus are sufficient to sustain near normal locomotion (M. Beattie, Ohio State University College of Medicine). It is therefore not surprising that profound early behavioural deficits are followed by considerable recovery ${ }^{1}$. The situation is similar in spinal cord ischaemia where only a small fraction of neurones needs to be saved for the animal to be able to walk. Here, serotonin antagonists reduce both the propagation of ischaemic damage and the subsequent high mortality rate in rabbits (Justin Zivin, University of California, San Diego). By improving transmitter release of spared neurones with a catecholamine-

* Pharmacological Appraaches to the Treatment of Brain and Spinal Cord Injury Walferdange, Luxembourg 7-11 July 1986 rgic agonist it was possible to reduce discrimination learning deficits after large neocortical lesions in rats and cats (D. Feeney, University of New Mexico).

Although all these drug treatments aim to help the surviving neurones, there are other cells of the nervous system whose role in brain repair has long been neglected: the glia cells. It has always been assumed that these cells, especially astrocytes, are detrimental to brain repair because they form a barrier around the wound, thus preventing regeneration across the injury site. But the reputation of astrocytes is improving (Manuel NietoSampedro, University of California, Irvine). They have the unique ability to proliferate in response to trauma and provide an environment that can help the spared neurones to deal with the consequences of injury. For example, they buffer the neuronal environment by regulating ionic balances and binding excess excitatory amino acids. In addition, astrocytes release trophic factors that can enhance neuronal growth and survival ${ }^{2}$. Because the need for neurotrophic factors is not satisfied until several days after injury, the initial balance between neurotrophic and neurotoxic substances (such as free fatty acid metabolites and excitatory amino acids) is unfavourable and cells die within several days after the primary damage. Thus, pharmacological treatments are currently being tested that try to engineer a post-injury environment with high neurotrophic and low neurotoxic levels.

Two agents that have now been tested extensively are nerve growth factor ${ }^{1.3}$ and gangliosides (glycolipids) ${ }^{4}$. Although it has been assumed that nerve growth factor and gangliosides enhance regeneration in the brain, their behavioural effects now seem to be caused by their ability to reduce secondary neuronal degeneration. It is believed that nerve growth factor is a trophic factor itself, whereas gangliosides may function as a potentiator of endogenous trophic factors. Intracerebral injections of nerve growth factor enhance neuronal survival in the septum after damage to the choli-

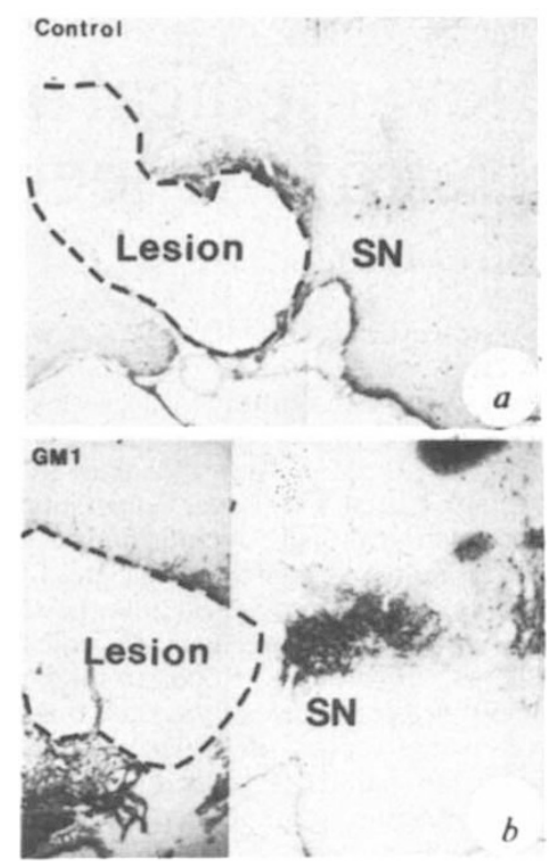

After transections of the nigrostriatal pathway, neurones near the lesion loose their axons and subsequently degenerate. Note the absence of cell labelling in the substantia nigra (SN) as seen here in a cross-section through the brain $(a)$. When treated daily with GM1 ganglioside, the survival of these neurones is significantly improved. $b$. Dopamine-containing neurones (dark area) shown by tyrosine hydroxylase immunocytochemistry (courtesy of Drs Toffano and Consolazione).

nergic system by fimbria-fornix transections ${ }^{3}$. Similarly, systemic injections of purified gangliosides reduce behavioural deficits after transections of the nigrostriatal pathway ${ }^{4}$ (see figure). Because they act by rescuing dying neurones, perhaps via a reduction of cerebral oedema ${ }^{5}$, the positive effects of these drugs depend on the presence of some spared neurones ${ }^{6}$.

Rather than enhancing regeneration of axons, drugs help the remaining neurones to survive. Because these neurones are already committed to the function impaired by the lesion, their salvation by drug treatment appears to be the most fruitful approach to reduce the devastating behavioural deficits seen after brain or spinal cord injury.

1. Finger, S. \& Stein, D. (j. Brain Dumage and Recovery (Academic, New York, 1982)

2. Nieto-Sampedro. M. \& Cotman, C.W. in Synaptic Plasticity (ed. Cotman, C.W.) 407 (Guilford, New York. 1985).

(ed. Cotman, C.W.) 407 (Guilford,

4. Sabel, B.S. et al. in Brain Plasticity, Learning and Memory (eds Will, B. et al.) 481 (Plenum, New York, 1985).

5. Karpiak, S.E. \& Mahadik, S.P. J. neurosci. Res. 12. 485 (1984)

6. Toffano, G. et al. J. neurosci Res. 12. 397 (1984)

Bernhard A. Sabel is at the Institute of Medical Psychology. University of Munich Medical School, Goethestr. 31, 8000 Munich, FRG and Donald G. Stein is at the Department of Psychology, Clark University, Worcester, Massachusetts 01610, USA. 